8. Ramos RL, Teixeira LA, Ormonde LR, et al. Emergence of mupirocin resistance in multiresistant Staphylococcus aureus clinical isolates belonging to Brazilian epidemic clone III: B:A. J Med Microbiol. 1999;48(3):303-7.

9. Clinical and Laboratory Standards Institute. Performance for antimicrobial susceptibility testing. $26^{\text {th }}$ ed. Wayne, PA: CLSI; 2016.

10. Ojeda-Sana AM, Repetto V, Moreno S. Carnosic acid is an efflux pumps modulator by dissipation of the membrane potential in Enterococcus faecalis and Staphylococcus aureus. World J Microbiol Biotechnol. 2013;29(1):137-44.

11. Xu Z, Liang Y, Lin S, et al. Crystal Violet and XTT Assays on Staphylococcus aureus Biofilm Quantification. Curr Microbiol 2016;73(4):474-82.

12. Günther F, Blessing B, Tacconelli E, Mutters N. MRSA decolonization failure-are biofilms the missing link? Antimicrob Resist Infect Control. 2017;6:32.

\title{
Anticuerpos antitransglutaminasa tisular IgA y contra péptidos de gliadina desaminados IgG como predictores de enfermedad celíaca
}

\author{
$\operatorname{Ig} A$ anti-tissue transglutaminase antibodies and IgG antibodies against \\ deamidated gliadin peptides as predictors of celiac disease
}

\author{
Dr. Gonzalo Ortiz ${ }^{a}$ Dra. Gabriela Messere ${ }^{a}$, Dra. María del C. Toca ${ }^{a}$, Dra. Mirian Fioruccib, \\ Dr. Román Bigliardi ${ }^{a}$, Dr. Jorge Vidal ${ }^{a}$ y Dr. Ricardo Reynoso ${ }^{a}$
}

\section{RESUMEN}

Objetivo. Comparar el rendimiento de anticuerpos antitransglutaminasa $\operatorname{Ig} \mathrm{A}$ (anti-TG2 $\operatorname{Ig} \mathrm{A}$ ), antiendomisio $\operatorname{Ig} \mathrm{A}$ (EMA IgA) y antigliadina desaminada IgA/IgG (AGADGP IgA/IgG) para el diagnóstico de enfermedad celiaca.

Métodos. Estudio descriptivo en pacientes con enfermedad celíaca. Se dosaron anticuerpos: AGADGP (IgA/IgG), EMA IgA, anti-TG2 IgA y biopsia intestinal. Sexo: mujeres (61\%). Mediana de edad: 78,4 meses.

Resultados. Se incluyeron 136 niños; 108 presentaron AGADGP IgA elevado; 124, AGADGP IgG aumentado; 128, EMA IgA positivo; 130, anti-TG2 IgA aumentado. Cuatro de 6 pacientes con anti-TG2 IgA negativos tenían AGADGP IgG elevado.

La combinación de los anticuerpos AGADGP IgG + antiTG2 IgA tuvo una correlación positiva en 134 pacientes y la combinación AGADGP IgG + EMA fue positiva en 133 niños. Conclusión. Se demostró la buena especificidad y sensibilidad de EMA IgA, anti-TG2 IgA y AGADGP IgG. La combinación AGADGP IgG/anti-TG2 mostró sensibilidad del 98-99 \% y especificidad del $100 \%$. La elección de anti-TG2 y AGADGP IgG da excelentes resultados, con bajo costo y no depende del operador.

Palabras clave:enfermedad celiaca, anticuerpos, diagnóstico, niños.

a. Sección de Gastroenterología, Hepatología y Nutrición Infantil, Servicio de Pediatría.

b. Servicio de Laboratorio.

Hospital Nacional Alejandro Posadas.

Correspondencia:

Dr. Gonzalo Ortiz: ortizgonzalojavier@gmail.com

Financiamiento: Ninguno.

Conflicto de intereses: Ninguno que declarar.

Recibido: 6-9-2017.

Aceptado: 15-8-2018 http:/ / dx.doi.org/10.5546/ aap.2019.52

Texto completo en inglés:

http:/ / dx.doi.org/10.5546/ aap.2019.eng.52

Cómo citar: Ortiz G, Messere G, Toca MC, Fiorucci M, et al. Anticuerpos antitransglutaminasa tisular IgA y contra péptidos de gliadina desaminados IgG como predictores de enfermedad celiaca. Arch Argent Pediatr 2019;117(1):52-55.

\section{INTRODUCCIÓN}

La enfermedad celíaca (EC) es una enfermedad crónica inmunomediada, sistémica, precipitada por la ingestión de granos que contienen gluten, que afecta al intestino delgado de individuos genéticamente predispuestos. ${ }^{1}$

Los anticuerpos contra proteínas propias (autoanticuerpos) son marcadores de autoinmunidad; pueden ser anti-transglutaminasa (anti-TG2) o antiendomisio (EMA). ${ }^{2-4}$

El anticuerpo EMA es el más específico. Este test presenta una alta sensibilidad (90-98 \%) y especificidad, realizado por inmunofluorescencia indirecta (IFI) y observador dependiente, por lo cual se utiliza en el algoritmo diagnóstico como test confirmatorio.

El anti-TG2 se realiza por el método de ensayo por inmunoabsorción ligado a enzimas (enzymelinked immunosorbent assay; ELISA, por sus siglas en inglés), que es más objetivo y sencillo, y se recomienda como herramienta inicial en el algoritmo diagnóstico de la EC. ${ }^{2-4}$

Se ha demostrado que la sensibilidad del 
anticuerpo antitransglutaminasa (anti-TG2) baja en los pacientes con daño intestinal leve, que se observa en los individuos con baja ingesta de gluten y en familiares de celiacos. Otra población que puede presentar falsos negativos al utilizar autoanticuerpos (anti-TG2 y EMA) son los niños menores de 3 años, con baja respuesta autoinmune..$^{2,3}$ En este grupo de pacientes, la combinación del anti-TG2 y anticuerpos desaminados de gliadina IgG (AGADGP IgG) puede mejorar la detección de casos de $\mathrm{EC}^{2-5}$

La utilización de los test que miden los anticuerpos AGADGP mostró mejorar la eficacia del diagnóstico al aumentar la sensibilidad manteniendo la especificidad. ${ }^{6}$ La biopsia intestinal continúa siendo un criterio diagnóstico indiscutible de EC. ${ }^{6,7}$

El objetivo de nuestro estudio fue comparar el rendimiento de anticuerpos anti-TG2 IgA, EMA IgA y AGADGP IgA/IgG para el diagnóstico de EC.

\section{MATERIAL Y MÉTODOS}

Estudio descriptivo y observacional, desde enero de 2011 a diciembre de 2014. Se incluyeron los pacientes que consultaron a la Sección de Gastroenterología Infantil, del Servicio de Pediatría del Hospital Nacional A. Posadas, con sospecha de EC para realizar una videoendoscopía digestiva alta (VEDA).

A todos los niños se les realizó el dosaje de IgA total y estudios serológicos: AGADGP IgA/IgG con método ELISA (QUANTA Lite Celiac), con valor de corte > 20 UI; EMA IgA con método de IFI considerado positivo con una dilución $>1: 5, y$ anti-TG2-IgA con método de ELISA (QUANTA), cuyo valor de corte fue $>20 \mathrm{U} / \mathrm{L}$.

A todos los pacientes se les realizó una VEDA y se tomaron seis biopsias (4 en la segunda porción del duodeno y 2 en el bulbo duodenal).

Los resultados del examen histopatológico fueron expresados de acuerdo con la clasificación de Marsh Oberhuber: Marsh 0 representaba la mucosa normal; Marsh 1, el incremento de linfocitos intraepiteliales (LIES); Marsh 2, el aumento de LIES con hiperplasia críptica, y Marsh 3, atrofia vellositaria. Se consideró que padecían EC los pacientes que presentaban Marsh 2 y 3.

El protocolo fue sometido a la evaluación y a la aprobación por el Comité de Ética del Hospital Nacional Alejandro Posadas. A todos los padres o tutores se les solicitó el consentimiento informado, y a los mayores de 12 años, el asentimiento.

Datos estadísticos: Se midió la sensibilidad, la especificidad, el valor predictivo positivo (VPP) y el valor predictivo negativo (VPN) de AGADGP IgA/IgG, EMA IgA, anti-TG2 IgA y las combinaciones de AGADGP IgG/anti-TG2 IgA y EMA IgA/ AGADGP IgG.

Todas las mediciones se realizaron con un intervalo de confianza del $95 \%$. Se utilizaron test de chi $^{2}$, curva de característica operativa del receptor (receiver operating characteristic; ROC, por sus siglas en inglés) con un intervalo de confianza del $95 \%$.

\section{RESULTADOS}

Fueron incluidos, durante un período de cuatro años, 136 niños. De ellos, 83 (61 \%) eran mujeres, mientras que $53(39 \%)$ eran varones. La edad mediana del total de los pacientes fue de 78,4 meses (con un rango de 12-192 meses). Ningún niño presentó déficit de IgA total.

La presentación clínica más frecuente fue, en 83 niños (61\%), clásica; la segunda fue la no clásica en 36 niños (26\%), y los 17 pacientes restantes $(13 \%)$ fueron asintomáticos. En este último grupo, todos presentaron más de un anticuerpo positivo.

La biopsia duodenal fue confirmatoria para EC en los 136 niños. Dos pacientes presentaron Marsh 2, y 134, Marsh 3 (102: 3 c; 29: 3 b; y 3: 3 a).

TABLA 1. Sensibilidad y especificidad de los test serológicos

\begin{tabular}{lcccc}
\hline Pacientes & AGADGP IgA & AGADGP IgG & EMA IgA & Anti-TG2 IgA \\
\hline Celíacos: 136 & $108(+) / 28(-)$ & $124(+) / 12(-)$ & $128(+) / 8(-)$ & $130(+) / 6(-)$ \\
Sensibilidad & $79 \%$ & $91 \%$ & $94 \%$ & $96 \%$ \\
Especificidad & $97 \%$ & $97 \%$ & $100 \%$ & $100 \%$ \\
VPP & $98 \%$ & $98 \%$ & $100 \%$ & $100 \%$ \\
VPN & $67 \%$ & $82 \%$ & $87 \%$ & $90 \%$ \\
\hline
\end{tabular}

AGA: anticuerpo antigliadina; DGP: proteína de gliadina desaminada; EMA: anticuerpo antiendomisio; anti-TG2: anticuerpo antitransglutaminasa; VPP: valor predictivo positivo; VPN: valor predictivo negativo; IgA: inmunoglobulina A; IgG: inmunoglobulina G. 
Con respecto a los resultados de los test serológicos, se observó una especificidad del $100 \%$ en los anticuerpos EMA IgA y anti-TG2 IgA, con el $97 \%$ en los anticuerpos AGADGP. La sensibilidad fue alta en EMA IgA y anti-TG2 (94$96 \%$, respectivamente), moderada en AGADGP $\operatorname{IgG}(91 \%)$ y baja en AGADGP IgA (79 \%). Véase la Tabla 1.

Se observó, como dato relevante, que, utilizando la combinación de 2 anticuerpos AGADGP IgG + EMA IgA y / o AGADGP IgG + anti-TG2 IgA, mejoraba la sensibilidad al 98-99\% y la especificidad al $100 \%$. Véase la Tabla 2.

En la población de los niños menores de 3 años (n: 39), se constató una baja sensibilidad de los test serológicos; fue del $90 \%$ en la anti-TG2 IgA. Los 4 pacientes con anti-TG2 normal presentaron AGADGP IgG elevado. Todos presentaron sintomatología clásica. Véase la Tabla 3.

\section{DISCUSIÓN}

Los test más sensibles y específicos son aquellos que detectan autoanticuerpos anti-TG2 y EMA, con una sensibilidad del 90-98 \% y una especificidad del 95-98\% en pacientes celíacos no tratados, sintomáticos y con grave daño de la mucosa. ${ }^{8}$

En los últimos años, ha sido recomendada la utilización de test (ELISA) que miden antiTG2 junto con AGADGP, con el fin de mejorar la sensibilidad. Ambos anticuerpos AGADGP IgA/ IgG demostraron tener mejor sensibilidad que los antigliadina (AGA clásicos) y, especialmente, los IgG, con mejor especificidad en pacientes pediátricos, de utilidad en pacientes con déficit de $\operatorname{Ig} A .^{7-9}$

En este estudio, la sensibilidad de AGADGP IgG (91\%) fue comparable a la de anti-TG2 (96 $\%$ ) y EMA (94\%); en cambio, AGADGP IgA tuvo una sensibilidad menor del $71 \%$. Con el fin de mejorar el rendimiento de los anticuerpos, se combinaron 2 de ellos: con EMA / AGADGP IgG, la sensibilidad mejoró al $98 \%$, con VPN del 95 $\%, \mathrm{y}$, cuando se combinaron anti-TG2/ AGADGP IgG, mejoró aún más la sensibilidad al $99 \%$, con VPN del $97 \%$.

Cuando se analizaron los resultados (los falsos negativos) que disminuyeron la sensibilidad de los test serológicos, teniendo en cuenta las edades de los pacientes con sospecha de EC, 5 de los 8 con EMA negativo y los 6 con anti-TG2 normales fueron niños menores de 3 años. La suma de alguno de estos anticuerpos con AGADGP IgG mejoró la sensibilidad en este grupo de edad para el diagnóstico de EC.

En cuanto a la especificidad, fue del $100 \%$ con VPP del $100 \%$ para los anticuerpos anti-TG2 y EMA, y del $97 \%$ para AGADGP IgA/IgG con

TABLA 2. Sensibilidad y especificidad de la combinación de los test serológicos

\begin{tabular}{lcc}
\hline Pacientes & EMA + AGADGP IgG & Anti-TG2 IgA + AGADGP IgG \\
\hline Celíacos: 136 & $133(+) / 3(-)$ & $134(+) / 2(-)$ \\
Sensibilidad & $98 \%$ & $99 \%$ \\
Especificidad & $100 \%$ & $100 \%$ \\
VPP & $100 \%$ & $100 \%$ \\
VPN & $95 \%$ & $97 \%$ \\
\hline
\end{tabular}

AGA: anticuerpos antigliadina; DGP: proteína de gliadina desaminada; EMA: anticuerpo antiendomisio; anti-TG2: anticuerpo antitransglutaminasa; VPP: valor predictivo positivo; VPN: valor predictivo negativo; IgA: inmunoglobulina A; IgG: inmunoglobulina G.

TABLA 3. Sensibilidad y especificidad de los estudios serológicos en pacientes menores de 3 años

\begin{tabular}{lcccc}
\hline Menores de 3 años (n: 39) & Sensibilidad & Especificidad & VPP & VPN \\
\hline AGADGP IgA (n: 29) & $74 \%$ & $100 \%$ & $100 \%$ & $55 \%$ \\
AGADGP IgG (n: 33) & $85 \%$ & $100 \%$ & $100 \%$ & $67 \%$ \\
EMA IgA (n: 34) & $87 \%$ & $100 \%$ & $100 \%$ & $68 \%$ \\
anti-TG2 IgA (n: 35) & $90 \%$ & $100 \%$ & $100 \%$ & $75 \%$ \\
\hline
\end{tabular}

AGA: anticuerpo antigliadina; DGP: proteína de gliadina desaminada; anticuerpos EMA: anticuerpo antiendomisio; anti-TG2: anticuerpo antitransglutaminasa; IgA: inmunoglobulina A; IgG: inmunoglobulina G; VPP: valor predictivo positivo; VPN: valor predictivo negativo. 
un VPP del $98 \%$. La especificidad del anticuerpo AGADGP IgG mejoró notablemente con respecto a la observada en estudios previos del anticuerpo AGA IgG clásico, el $97 \%$ versus el $63 \%$. La combinación de 2 anticuerpos EMA / AGADGP IgG o anti-TG2 / AGADGP IgG mostró una alta especificidad, que fue, en ambos casos, del $100 \%$ y un VPP del $100 \%$.

En nuestro estudio, se observó que los anticuerpos AGADGP IgG presentaron mayor sensibilidad y especificidad que los IgA. Como lo detallan Prause y col., el rendimiento de los anticuerpos de tipo AGADGP IgG superaría el de los de clase IgA, sobre todo, en aquellos pacientes con déficit de IgA y en menores de 2 años. En los primeros años de vida, la TG2 puede ser falsamente negativa o con marcada fluctuación en sus niveles, debido a que su mecanismo de generación inmunológica sería más lento, con elevación de los títulos recién de 1 a 2 años después de la exposición al gluten. ${ }^{10-12}$

En nuestro estudio, durante este período, no hubo pacientes con déficit de IgA, pero hay varias publicaciones que muestran la buena sensibilidad y especificidad del anticuerpo AGADGP IgG en esta población de riesgo, lo que lo hace recomendable para el estudio diagnóstico de la EC. ${ }^{10-12}$

\section{CONCLUSIONES}

Con nuestro estudio, se confirmó la buena especificidad y sensibilidad de los test que detectaban anticuerpos EMA, anti-TG2 y AGADGP IgG, y la baja sensibilidad del AGADGP IgA. La suma de los test serológicos, AGADGP IgG con EMA y anti-TG2, mostró una sensibilidad del $98 \%$ al $99 \%$ y una especificidad del $100 \%$. La elección de anti-TG2 y AGADGP IgG da excelentes resultados, con bajo costo y no depende del operador.

\section{REFERENCIAS}

1. Ludvigsson JF, Leffler DA, Bai JC, Biagi F, et al. The Oslo definitions for coeliac disease and related terms. Gut. 2013; 62(1):43-52.

2. Fasano A, Araya M, Bhatnagar S, Cameron D, et. al. Federation of International Societies of Pediatric Gastroenterology, Hepatology, and Nutrition Consensus Report on Celiac Disease. J Pediatr Gastroenterol Nutr. 2008; 47(2):214-9.

3. Aleanzi M, Demonte A, Esper C, Garcilazo S, et.al. Celiac Disease: Antibody recognition against native and selectively deaminated gliadin peptides. Clin Chem. 2001;47(11):2013-28.

4. Murch S, Jenkins $H$, Auth $M$, Bremmer $R$, et al. Joint BSPGHAN and Coeliac UK guidelines for the diagnosis and management of coeliac disease in children. Arch Dis Child. 2013; 98(10):806-11.

5. Kupfer S, Jabri B. Pathophysiology of celiac disease. Gastrointest Endosc Clin N Am. 2012; 22(4):639-60.

6. Volta U. Coeliac Disease: Time for a new diagnostic approach in symptomatic children. J Pediatr Gastroenterol Nutr. 2013; 56(3):241.

7. Rubio-Tapia A, Hill ID, Kelly CP, Calderwood A, et al. ACG clinical guidelines: diagnosis and management of celiac disease. Am J Gastroenterol. 2013;108(5):656-76.

8. NiveloniS, Sugai E, Cabanne A, Vazquez H, etal. Antibodies against synthetic deamidate gliadin peptides as predictors of celiac disease: Prospective assessment in an adult population with a high pretest probability of disease. Clin Chem. 2007; 53(12):2186-92.

9. Vermeersch P, Geboes K, Mariën G, Hoffman I, et al. Serological diagnosis of celiac disease: Comparative analysis of different strategies. Clin Chim Acta. 2012;413(2122):1761-7.

10. Giersiepen K, Lelgemann M, Stuhldreher N, Ronfani L, et al. Accuracy of Diagnostic Antibody Tests for Coeliac Disease in Children: Summary of an Evidence Report. J Pediatr Gastroenterol Nutr. 2012; 54(2):229-41.

11. Husby S, Koletzko S, Korponay-Szabó IR, Mearin ML, et al. European Society for Pediatric Gastroenterology, Hepatology, and Nutrition guidelines for the diagnosis of coeliac disease. J Pediatr Gastroenterol Nutr. 2012; 54(1):136-60.

12. Bufler G, Heilig G, Ossiander F, Freudenberg V, et al. Diagnostic performance of three serologic tests in childhood celiac disease. Z Gastroenterol. 2015; 53(2):108-14. 